\title{
Percepção da Equipe de Enfermagem Quanto à Assistência Provida em uma Unidade de Internação
}

\author{
Perception of the Nursing Team Regarding the Assistance \\ Provided in a Hospitalization Unit
}

\author{
MARISTELA OLIVEIRA LARA ${ }^{1}$ \\ FERNANDO PEREIRA CHAVES ${ }^{2}$ \\ EMERSON VINÍCIUS OLIVEIRA BRAGA ${ }^{3}$ \\ DULCE APARECIDAMARTINS ${ }^{1}$ \\ JOSÉ CRISTIANO RAMOS GLÓRIA ${ }^{4}$ \\ PATRÍCIAWICHR ${ }^{5}$
}

\section{RESUMO}

Introdução: A enfermagem, dentro dos serviços de saúde, é considerada fundamental na atenção ao paciente. Assim, sua visão sobre a prática executada é necessária como componente de avaliação de qualidade da assistência e reflexão sobre a prática. Objetivo: Compreender a percepção da equipe de enfermagem sobre os cuidados providos em uma unidade de clínica médica de um hospital de referência macrorregional em saúde do interior do estado de Minas Gerais. Material e Métodos: A metodologia utilizada foi o estudo de caso, abordagem qualitativa, com entrevistas semiestruturadas. Foram entrevistados 11 profissionais de enfermagem (Técnicos e Enfermeiros), funcionários a pelo menos um ano no local do estudo. Utilizou-se a Análise de Conteúdo Temático para interpretação dos dados e posterior categorização temática. Resultados: A análise dos dados revelou que aequipe de enfermagem possui um bom relacionamento interpessoal, reconhece a necessidade de capacitação e maior humanização nas ações como requisitos para a melhoria da assistência prestada. Enfatizam o papel e a postura do enfermeiro como norteador na condução do processo de trabalho da equipe. Por outro lado, as entrevistas revelaram que existem dificultadores (recursos humanos e materiais insuficientes, desorganização do processo de trabalho) para que se possa prestar uma assistência satisfatória. Conclusão: A percepção da equipe de enfer-magem sobre os cuidados providos revela potencialidades relacionadas a interdicisplinaridade, autoavaliação positiva e deficiências em relação a capacitações, humanização e processo de trabalho.

DESCRITORES

Enfermagem. Capacitação Profissional. Qualidade da Assistência à Saúde.Serviço Hospitalar de Enfermagem. Gestão de Qualidade.

\begin{abstract}
Introduction: Among the healthcare services, nursing is considered to fundamental for patient care. Thus, the view of nursing professionals on their practice performance is needed as a component of quality assessment of careand reflection on practice. Objective: To understand the perception of the nursing team about the care provided in a medical clinic unit of a macro-regional referral hospital in the interior of the state of Minas Gerais. Material and Methods: This was a case study, with a qualitative approachusingsemi-structured interviews. Eleven nurses (technicians and nurses) were interviewed, who were employees for at least one year at the study site. Thematic content analysis was used to interpret the data and, later, thematic categorization. Results: The data analysis revealed that the nursing team has a good interpersonal relationship, recognizes the need for training and greater humanization in the actions as requirements for the improvement of care provided. They emphasize the role and the position of the nurse as guiding in the conduction of the work process of the team. On the other hand, the interviews revealed that there are difficulties (insufficient human and material resources, disorganization of the work process) in order to provide satisfactory assistance. Conclusion: The nursing team's perception about the care provided reveals multidisciplinary potentialities, positive self-assessment and deficiencies in relation to training, humanization and work process.
\end{abstract}

\section{DESCRIPTORS}

Nursing.Professional Training.Quality of Health Care.Nursing Service Hospital.Quality Management.

1 Professora Adjunta do Departamento de Enfermagem da Universidade Federal dos Vales do Jequitinhonha e Mucuri - UFVJM. Diamantina. Minas Gerais. Brasil.

2 Enfermeiro. Hospital Margarida. João Monlevade. Minas Gerais. Brasil.

3 Enfermeiro. Responsável Técnico do Hospital Nossa Senhora da Saúde - HNSS. Diamantina. Minas Gerais. Brasil.

4 Professor Adjunto do Departamento de Odontologia da Universidade Federal dos Vales do Jequitinhonha e Mucuri - UFVJM. Diamantina. Minas Gerais. Brasil.

5 Professora de Enfermagem do Centro Universitário Unifafibe. Bebedouro. São Paulo. Brasil. 
A $s$ transformações ocorridas no mundo moderno, novas tecnologias, aumento do conhecimento científico, interferências da mídia, entre outras, tem feito com que as empresas dos diversos setores se adaptem ao novo cenário. Os serviços de saúde, sendo considerados empresas prestadoras de cuidadosnão estão isentos destas mudanças, assim faz-se necessário a atuação profissional e a criação de novas estratégias capazes de atender ao seu cliente de forma holística, prestando assistência de qualidade ${ }^{1,2}$. Nesse contexto, a enfermagem se depara com a necessidade de aprimorar seus processos de trabalho com vistas à garantia de cuidados com qualidade aos pacientes ${ }^{3}$.

A enfermagem é considerada fundamental na atenção ao paciente, tanto na identificação e no atendimento as necessidades de cuidados, quanto pelo seu vínculo com o cliente ${ }^{4}$. O cuidado humanizado deve ser sempre um aspecto importante no trabalho da enfermagem e fundamental no reestabelecimento da saúde dos pacientes ${ }^{1}$. Dessa forma, um avanço no que diz respeito à compreensãoda assistência que é prestada por esses profissionais faz-se prudente para que os usuários tenham um atendimento que satisfaça suas necessidades².

Aprestação de cuidadosem saúde adquiriu significado particular quanto ao atendimento ao cliente, sendo constituído por atributos que envolvem a excelência profissional e um alto grau de satisfação por parte dos usuários, internos e externos. Assim, avaliar a qualidade da atenção não é uma tarefa fácil, porém, seus resultados apoiam os gestores na tomada de decisão e a identificação de oportunidades de melhoria e reajuste de metas ${ }^{5}$.

Para averiguar como tem sido a assistência de enfermagem dentro das instituições de saúde, é necessário uma avaliação dos serviços prestados, buscando dessa forma apropriar-se da visão não só dos usuários ${ }^{2,4}$, mas também dos executores dos cuidados ${ }^{4}$. É importante saber a visão daqueles que estão inseridos no processo de trabalho, sobre a percepção dos mesmos em relação aos fatores que irão interferir nesse processo ${ }^{2}$.

Estudos de percepção da enfermagem já vem sendo empregados para gestão de qualidade total e com um retorno importante para as instituições hospitalares ${ }^{5}$. Tal avaliação não deve ser vista como forma punitiva nem mesmo restritiva para o serviço, mas para que através dos dados levantados o enfermeiro possa traçar metas e objetivos a serem alcançados em busca de melhorias no atendimento prestado pela categoria profissional, uma vez que a busca pela excelência da assistência, apesar de ser uma tarefa árdua, deve ser algo cotidiano na vida de cada profissional de saúde ${ }^{2}$.

Sob esse aspecto, o estudo teve como objetivo compreender a percepção da equipe de enfermagem sobre os cuidados providos em uma unidade de clínica médica de um hospital de referência macrorregional em saúde do interior do estado de Minas Gerais. Estudos com esse enfoque são importantes, pois possibilitam identificar os obstáculos e possíveis problemas na assistência prestada, podendo assim estabelecer estratégias para melhorias no atendimento.

\section{MATERIAL E MÉTODOS}

Investigação do tipo estudo de caso com abordagem qualitativa. Este método tem a capacidade de retratar a realidade de forma mais completa e profunda sem a pretensão de generalizar o fenômeno, além de apontar a multiplicidade e as dimensões do problema e dá ao leitor a oportunidade de fazer suas próprias inferências e construir novos significados ${ }^{6,7}$.

Como instrumento de coleta de dados foi utilizada entrevista semi-estruturada, que é o procedimento mais usual no trabalho de campo, pois possibilita obter informações esclarecedoras por meio das falas dos sujeitos sociais ${ }^{8}$.

A entrevista representa um instrumento privilegiado de coleta de informações, uma vez que a fala é reveladora das condições estruturais, dos sistemas de valores, normas e símbolos e das condições históricas, socioeconômicas e culturais de uma população. Oferece ainda, todas as possibilidades para que o informante alcance a liberdade e a espontaneidade necessárias para que sua participação contribua para o enriquecimento da investigação ${ }^{8}$.

As entrevistas foram realizadas de maio a junho de 2010 com a equipe de enfermagem que presta assistência na Clínica Médica de um hospital geral do interior de Minas Gerais, referência para macrorregião em saúde. A clientela do Sistema Único de Saúde corresponde a $90 \%$ das hospitalizações, os demais são clientes de convênios e particulares.

As entrevistas foram gravadas e em seguida transcritas, na íntegra, para se preservar a fidedignidade das informações. As questões nortea- 
doras da entrevista foram: 'Descreva o relacionamento da enfermagem com os demais profissionais da saúde'; 'Qual sua percepção quanto a capacitação da equipe de enfermagem?'; 'Quais são as maiores dificuldades que a enfermagem enfrenta para a prestação dos seus cuidados?'; 'O que pode ser melhorado que está no seu alcance?'; 'Como você classifica o serviço de enfermagem? Justifique'; 'Qual a sua visão em relação ao papel do enfermeiro na qualidade da assistência?'.

Tendo em vista que a pesquisa qualitativa não tem por finalidade a generalização, o critério dessa forma não é numérico. Sendo assim, o pesquisador deve preocupar-se em buscar os sujeitos com atributos e orientá-los para o objetivo em questão. Sendo assim, o número de sujeitos pesquisados obedeceu à técnica de saturação de dados, que consiste na reincidência das informações, muitas vezes não acrescentando ao objetivo da pesquisa ${ }^{8}$. Dessa forma, foram entrevistados 11 profissionais de enfermagem (Técnicos e Enfermeiros), funcionários a pelo menos um ano no local do estudo, sendo a escolha dos mesmos de forma aleatória simples, por sorteio e de acordo com a disponibilidade no período da coleta de dados.

Para a análise e interpretação dos dados foi utilizada a Análise de Conteúdo Temática, que consiste em descobrir os núcleos de sentido que compõem uma comunicação cuja presença ou frequência tem um significado para o objetivo analítico visado ${ }^{8}$. A operacionalização desse estudo seguiu as fases da análise Temática, com leituras flutuantes de cada entrevista buscando apropriarse do conteúdo e em seguida, leituras exaustivas, com o objetivo de relacionar os pontos convergentes e divergentes entre as falas e extrair os temas relevantes que emergiram dos discursos dos entrevistados no sentido de alcançar a categorização temática. Após a categorização dos dados seguiuse com o tratamento e interpretação dos mesmos, trabalhando com a análise dos seus significados.

Os aspectos éticos foram respeitados, e a pesquisa foi aprovada pelo Comitê de Ética em Pesquisa da Universidade Federal dos Vales do Jequitinhonha e Mucuri (UFVJM) sob o Protocolo $n^{\circ}$ 042/2010e realizada de acordo com a Resolução n466/12do Conselho Nacional de Saúde para pesquisas com seres humanos, no que diz respeito ao sigilo, anonimato, consentimento livre e esclarecido e liberdade de desistir a qualquer momento da pesquisa. Além disso, foi solicitada a autorização do hospital para a realização do estudo.
Para apresentar a visão dos sujeitos quanto à temática, os mesmos foram codificados, no momento de suas falas, com a letra $F$ (funcionário) seguida de um algarismo (F1, F2, F3...).

\section{RESULTADOS E DISCUSSÃO}

A análise e interpretação dos dados permitiram a construção de cinco categorias temáticas que emergiram do discurso dos sujeitos dessa pesquisa, sendo elas: "Relacionamento entre os membros da equipe multiprofissional"; "Percebendo a capacitação profissional"; "Fatores dificultadores da assistência"; "Qualificando a assistência de enfermagem - a importância da humanização" e "A compreensão da equipe quanto ao papel do enfermeiro". Essas categorias encontram-se a seguir:

\section{Relacionamento entre os membros da equipe multiprofissional}

Sabe-se hoje, da grande importância do atendimento e trabalho multiprofissional em saúde, onde os membros da equipe reúnem esforços para assistir aos indivíduos em suas necessidades. 0 processo de trabalho exige dos trabalhadores a convivência com distintos saberes e poderes peculiares às relações interprofissionais, direcionados à alteridade ${ }^{9}$.

O trabalho em equipe tem como um dos objetivos a obtenção de impacto sobre os diferentes fatos que interferem no processo saúde/doença. A atuação multiprofissional deve ser interdisciplinar ou transdisciplinar, que consiste em fornecer a possibilidade da prática de um profissional se reconstruir na prática do outro, ambas sendo transformadas para a intervenção ${ }^{9,10}$. Nesse contexto, é relevante que os profissionais que compõem a equipe multiprofissional tenham um bom relacionamento e consigam estabelecer projetos assistenciais compartilhados ${ }^{10}$.

Foi percebido dificuldades de estabelecer um bom relacionamento por pequena parte dos entrevistados, como demonstrado na fala de F6:

“...pessoas que estão aqui só pra procurar nossos erros, nunca seus acertos, nunca sabem passar um elogio, nunca querem saber como é que foi o seu plantão, o seu dia, só procura saber o que você fez de errado para te entregar pra supervisão...". (F6) 
Ao se fazer uma análise desse discurso, percebe-se a dificuldade que essa equipe tem para atuar juntos em prol da troca de informações e utilização de técnicas para uma abordagem apropriada ao cliente. A busca da integralização está condicionada a uma recusa ao reducionismo e à objetivação dos sujeitos e consequentemente a abertura para o diálogo ${ }^{10}$. Em contrapartida, o discurso de outros, revelam possuírem um relacionamento bom, amigável e profissional com todos os membros da equipe multiprofissional que presta serviços na clínica médica. Isso pode ser confirmado quando F4 diz que:

"É um relacionamento profissional... nos relacionamos com profissionais médicos, fisioterapeutas, psicólogos, nutricionistas, as meninas da copa, o pessoal da limpeza. É um relacionamento bom, harmonioso..."

Para que se alcancem os reais objetivos do trabalho multiprofissional, faz-se necessário que os membros da equipe somem seus conhecimentos específicos em prol da qualidade do atendimento provido aos pacientes. Nesse contexto, não basta que os especialistas em saúde tenham domínio e apliquem isoladamente seus saberes profissionais específicos, é preciso somar saberes para dar respostas efetivas e eficazes aos problemas complexos que envolvem as perspectiva de viver com qualidade, incluindo o ambiente de trabalho ${ }^{10}$.

Portanto, o grande desafio do trabalho em equipe e da relação entre todos os profissionais, consiste em pensar no cuidado à saúde como uma relação de complementaridade e interdependência, que visa o atendimento humanizado e de qualidade aos clientes em situação de hospitalização ${ }^{11}$.

\section{Percebendo a capacitação profissional}

Atualmente, pela análise do mercado de trabalho, percebe-se, a capacitação profissional como um requisito básico para a atuação nos diferentes campos de trabalho. No setor saúde não é diferente, haja vista que as instituições estão à procura de recursos humanos cada vez mais capacitados para assumirem algum cargo ${ }^{12}$.

A noção de competência e capacitação profissional possibilita o desenvolvimento de enfermeiros pensantes, capazes de reflexão/ação social crítica e de opção pela postura de sujeitos construtores do conhecimento na prática profissional da enfermagem, nos diversos âmbitos e lugares sociais do trabalho ${ }^{13}$.

Neste estudo, os sujeitos apontaram a importância da capacitação profissional como um dos requisitos para melhoria da assistência prestada pela equipe de enfermagem, ressaltando a relevância da educação continuada como sendo uma forma de adquirir conhecimento necessário para a prática diária da enfermagem.

“...ultimamente nós estamos tendo bastante treinamentos, é muita informação que até pouco tempo atrás agente não tinha tanto, agora melhorou bastante". (F3)

Ao analisar o discurso anterior pode-se inferir que as instituições estão se preocupando cada vez mais com a capacitação dos seus funcionários, haja vista que hoje as educações continuadas fazemse frequentes nas instituições. Esse método tem objetivo de adequar os profissionais de enfermagem ao trabalho na respectiva unidade, para uma atuação orientada por conhecimentos e comportamentos institucionalizados e pré-estabelecidos ${ }^{13}$. Já a educação permanente é uma estratégia para que o indivíduo tenha mais habilidade e oportunidade de construir-se dentro do mundo do trabalho, auto reflexiva, em um movimento dinâmico e complexo mediado por valores políticos, socioculturais e éticos ${ }^{12,13}$.

O próximo relato contrapõe o anterior quando um sujeito se refere aos enfermeiros e aponta a necessidade mais capacitações:

"o pessoal tem que ter um treinamento melhor, acho que eles ainda estão muito crus, muito devagar... aqui dentro não é só teoria, a prática fala muito mais alto." (F5)

Na percepção dos sujeitos do estudo, para a melhoria da assistência, é necessário que os profissionais se capacitem cada vez mais, tanto no ambiente externo à instituição, quanto nas estratégias de capacitação fornecidas pelo hospital. Haja vista que profissionais mais bem capacitados poderão fornecer uma assistência mais resolutiva para os seus clientes ${ }^{12}$.

\section{Fatores dificultadores da assistência}

$\mathrm{Na}$ assistência à saúde, a Organização Mundial de Saúde, remete aos elementos: alto grau de competência profissional, a eficiência na utilização dos recursos, um mínimo de riscos, um 
alto grau de satisfação dos pacientes e um efeito favorável na saúde. Para uma assistência de qualidade, os profissionais de saúde devem combinar sua competência individual, responsividade e recursos ofertados pela instituição em que estão inseridos ${ }^{12}$.

No transcorrer desse estudo foi percebido que as grandes dificuldades apontadas pela equipe de enfermagem para o provimento do cuidado encontram-se no número limitado de recursos humanos na clínica médica, na escassez de recursos materiais e a desorganização no processo de trabalho (metodologia da assistência). As falas a seguir ilustram a visão dos funcionários quantos aos fatores dificultadores da assistência:

“...pra você prestar uma assistência melhor, o número de leitos para cada profissional é muito.... quando você está com a enfermaria cheia, você não tem tempo de prestar um serviço bacaninha". (F7).

“...os equipamentos que agente usa pra prestar o atendimento, muitas vezes a casa não tá podendo". (F6).

"O pessoal tem mania de reclamar sobre estrutura física, recursos materiais, mas acho que na verdade é o processo de trabalho que é conturbado. Então a principal dificuldade é agente conseguir que todos da equipe tenham uma visão de organização do processo de trabalho. (F4)

É importante ressaltar que a assistência provida nas instituições hospitalares a equipe de enfermagem tem uma grande expressão porque permanece com os clientes grande parte do tempo, promovendo a manutenção, reabilitação e a recuperação da saúde, através do cuidado constante ${ }^{14}$.

Entre os muitos desafios enfrentados pela enfermagem, um dos fatores preocupante, encontrase na falta ou em recursos humanos inadequados dentro das instituições, haja vista que a assistência de excelência depende diretamente dos envolvidos nesse processo. Apesar da alta carga de trabalho, muitos hospitais não obedecem ao percentual da equipe de enfermagem exigido nos padrões científicos e pelo Conselho Federal de Enfermagem, além das inúmeras não conformidades relacionadas ao cuidado, administração e educação continuada dos profissionais ${ }^{15}$.

Os aspectos qualitativos e quantitativos do pessoal de enfermagem requerem a atenção dos gestores de serviços da categoria, em virtude das implicações que o dimensionamento inadequado desses recursos causa sobre o resultado da assistência ${ }^{16}$.

Para que a prática de enfermagem possa obedecer aos princípios éticos da profissão, é necessário que as instituições que prestam serviços de saúde forneçam condições de trabalho para os profissionais, provendo os materiais que são necessários para o cuidado ao paciente. É sabido que o improviso de equipamentos/materiais devido à falta de recursos materiais prejudica a assistência, sendo que muitas vezes a falta de alguns materiais pode colocar os pacientes em situação de risco ou causar repercussões psicofísicas à equipe de trabalho ${ }^{17}$.

Quando o profissional atribui o processo de trabalho como fator dificultador da assistência, possibilita a reflexão quanto à função do enfermeiro dentro das instituições, pois a metodologia do cuidado está diretamente ligada à pessoa do enfermeiro como membro e supervisor da equipe, sendo esse o principal responsável pelo planejamento do trabalho da enfermagem dentro dos serviços de saúde. Dessa forma, pensar no cuidado é muito mais que apenas o atendimento às necessidades do paciente, mas também o planejamento dessa assistência, para que o mesmo possa ser realmente de excelência. O processo de enfermagem, como instrumento para assistência, possibilita a elaboração de uma prescrição de cuidados individualizados, além de viabilizar a melhoria nos registros e a humanização da assistência ${ }^{18}$.

\section{Qualidade da assistência de enfermagem - a importância da humanização}

A busca pela qualidade nos serviços de saúde tornou-se um fenômeno mundial, consequente do aumento da conscientização de que a satisfação é indispensável para a sobrevivência dos serviços de saúde. Além disso, constitui uma responsabilidade ética e social ${ }^{2,3}$.

Associando a qualidade da assistência de enfermagem com a humanização do atendimento, ter-se-á o que de fato é almejado pelos serviços de saúde, bem como para os clientes que todos os dias são atendidos nas instituições. Assim será possível atender aos três pilares propostos pelo Sistema Único de Saúde: Equidade, Universalidade e Integralidade ${ }^{19}$. 
Nesse enfoque de primazia da assistência e humanização, predominou entre os sujeitos entrevistados a descrição da atuação da equipe de enfermagem como sendo "boa", atendendo as necessidades individuais de cada paciente. Em sua fala, F4 traz a seguinte expressão que revela como tem sido o atendimento de enfermagem no referido hospital:

"apesar da gente não ter aqui indicadores de qualidade, assim científica, agente tem essa avaliação empírica. Eu classifico como um serviço bom, nós já conseguimos um progresso enorme".

Apesar da grande maioria dos entrevistados terem enfocado que no hospital em estudo a assistência de enfermagem tem sido de qualidade, mesmo com uma avaliação empírica, em contraponto, é importante revelar a fala do F5:

"o pessoal para abordar o paciente, a família, recepcionar, eu acho que falta muita coisa. Então assim, eu acho que a enfermagem não é só chegar e medicar, acho que começa desde a hora que você entrou ali, e isso falta muito".

A fala desse profissional proporciona uma reflexão sobre o verdadeiro atendimento humanizado, haja vista que assistência desejada ultrapassa a realização de procedimentos e técnicas no ambiente hospitalar. A humanização pode ser entendida como uma política que supõe necessariamente ultrapassar fronteiras, muitas vezes rígidas, dos diferentes saberes/poderes que se ocupam da produção da saúde. Dessa forma é possível entender que a assistência de enfermagem qualificada vai além da boa prática profissional ${ }^{19}$.

Um estudo da percepção dos pacientes no cenário deste estudo realizado na mesma época apontou $100 \%$ de satisfação com a enfermagem. Embora tenha identificado pacientes com postura de não exercício da autonomia, expectadores do cuidado e que não cobram por uma assistência humanizada, uma vez que pensam que simplesmente pelo fato de estar sendo tratado e medicado já é o suficiente. Ademais, revela o enfermeiro como um profissional ainda distante de suas ações privativas e sobrecarregado com várias atividades $^{2}$.

Para que a enfermagem possa alcançar níveis elevados, relacionados à assistência humanizada e qualificada, os profissionais devem além de possuírem conhecimento técnico científico, entender a dinâmica de interação com o cliente e sua família, com uma visão holística dos mesmos, dessa forma alcançando a satisfação da clientela que de fato são importantes na avaliação da qualidade ${ }^{20}$. Ressalta-se que a humanização é um conjunto de ações: comunicação, acolhimento, visão holística, gentileza, respeito ${ }^{1}$ e também condições adequadas de trabalho ${ }^{20}$.

\section{Compreensão da equipe quanto ao papel do enfermeiro}

A relevância do papel do enfermeiro na abordagem sobre a assistência foi enfatizada na concepção dos profissionais de enfermagem. É sabido que a enfermagem, dentro do cenário hospitalar exerce funções diversificadas, como o planejamento da assistência, cuidados propriamente dito, e questões burocráticas que são de sua competência ${ }^{12}$. Desta forma, os profissionais entrevistados ressaltaram a relevância do enfermeiro em lidar com a complexa rede que envolve a assistência ao paciente. $\mathrm{O}$ depoimento revela que:

"O Enfermeiro é essencial na qualidade da
assistência. Ele deve conduzir a equipe
para prestar os cuidados que aquele
paciente realmente precisa, e isso não é
nada mais, nada menos que a metodologia
do cuidado, do que o processo de
enfermagem..."(F4)

O relato anterior descreve de forma bem clara o papel que se espera do enfermeiro dentro de uma instituição como componente de uma equipe de trabalho. Quando o enfermeiro é capaz de planejar o seu trabalho de forma a executar sistematicamente o processo de enfermagem, espera-se que a assistência e a qualidade da mesma sejam percebidas com maior clareza, pois a metodologia dentro de sua essência proporciona um atendimento que vá de encontro às reais necessidades da clientela ${ }^{18}$.

Além dessa abordagem relacionada ao processo de trabalho, foi enfatizada pelos sujeitos a questão do enfermeiro na supervisão de enfermagem, onde muitas vezes os mesmos tem executado a postura de chefe e não de líder. As falas revelam essas questões: 
“...o enfermeiro tem de estar por dentro de tudo, mas estão formando enfermeiros só para mandar... Muitos formam com aquela ideiana cabeça, é pra mandar". (F6)

"eles tem de conscientizar que estão aqui para somar e isso na maioria das vezes não acontece. Um chefe não é um supervisor, eu acho que tem uma diferença muito grande principalmente na área da enfermagem". (F5)

Essas falas estimulam a reflexão acerca da postura do enfermeiro dentro das instituições, onde a postura de chefe tem sobreposto a de liderança. É por meio da liderança que o enfermeiro procura conciliar os objetivos organizacionais com os objetivos da equipe de enfermagem, buscando o aprimoramento da prática profissional e principalmente $o$ alcance de um trabalho efetivo $e$ integrador, cuja finalidade é a supremacia do cuidado $^{14}$. O depoimento seguinte enfatiza a questão da liderança:

“...eles tem que conscientizar que tem de partir deles para uma equipe funcionar...". (F5)

É suma importância, que os enfermeiros responsáveis pelo processo gerencial tenham sensibilidade para captar as necessidades emergenciais, habilidade para empreender e estimular ações inovadoras e flexibilidade para se adaptar às diferentes situações que se apresentam nas relações e interações do dia-a-dia das organizações do cuidado ${ }^{18}$. A promoção de uma boa assistência de enfermagem é favorecida quando os integrantes da equipe sentem-se satisfeitos no seu ambiente de trabalho e expressem a plena utilização de suas habilidades e potencialidades ${ }^{14}$.

Faz-se necessário, dentro desse enfoque, apresentar as principais ações do enfermeiro dentro das instituições, segundo relatos dos sujeitos da pesquisa: fiscalizar o serviço de enfermagem, realizar de procedimentos privativos dos mesmos, resolver questões burocráticas, coordenação eauxílio no serviço médico. Percebe-se que o enfermeiro exerce múltiplas atividades, e por vezes deixa de executar as que são realmente privativos de sua profissão, como mostra o seguinte relato:

“...o enfermeiro ele é secretário, conselheiro, ele é tudo... Se agente tiver o enfermeiro que tenha a visão do planejamento da assistência e que jamais o dever dele é de apagar fogo...". (F4)

Dessa forma é preciso que o enfermeiro reflita sobre o seu papel profissional, para que não se torne uma ação mecanicista e desprovida de perspectivas para o alcance da qualidade.

\section{CONCLUSÃO}

Compreender a percepção da equipe de enfermagem sobre os cuidados providos permitiu a identificação de potencialidades relacionadas a interdicisplinaridade, autoavaliação positiva e deficiências em relação a capacitações, humanização e processo de trabalho; todo esse conjunto influencia no nível de qualidade dos serviços prestados. Os achados contribuem para reflexões com a equipe, proporciona o conhecimento das necessidades e expectativas destes profissionais, subsidia as estratégias gerenciais do enfermeiro e da administração da instituição de saúde.

No que concerne à qualidade do cuidado prestado a equipe não classificou o mesmo, mas ponderou aspectos que reconhecem como primordiais de estar observando e aperfeiçoando para garantia de melhorias. Pode-se concluir que todos são elementos passíveis de transformação.A limitação do estudo está na análise de uma única unidade de internação, que pode apresentar características diferentes das demais.

\section{REFERÊNCIAS}

1. Carvalho DO, Santos NNRC, Silva ARV, Carvalho GCN. Percepção do profissional de enfermagem acerca do cuidado humanizado no ambiente hospitalar. R. Interd. 2015;8(3):61-74.

2. Braga EVO, Chaves FP, Lara MO, Wichr P, Martins DA, Glória JCR. O olhar do paciente frente à assistência de enfermagem em um hospital geral. Rev. enf-UFJF. 2016;2(1):21-9

3. Freitas JS, Silva AE, Minamisava R, Bezerra AL, Sousa MR. Qualidade dos cuidados de enfermagem e satisfação do paciente atendido em um hospital de ensino. Rev. latinoam. enferm. 2014;22(3):454460.

4. Martins PF, Perroca MG. Carenecessities: the view of the patient and nursing team. Rev. bras. enferm. 2017;70(5):1080-6. 
5. Vituri DW, Évora YDM. Total Quality Management and hospital nursing: anintegrativeliteraturereview. Rev. bras. enferm. 2015;68(5):945-52.

6. Cronin C. Using case study research as a rigorousformofinquiry. Nurse Res. 2014;21(5):19-27.

7. Coimbra MNCT, Martins AMO. O estudo de caso como abordagem metodológica no ensino superior. Nuances. 2014;24(3):31-46.

8. Minayo MCS. O desafio do conhecimento: pesquisa qualitativa em saúde. $14^{\circ}$ ed. São Paulo: Hucitec; 2014.

9. Van Bewer V. Transdisciplinarity in Health Care: A ConceptAnalysis. NursForum. 2017;52(4):339-47.

10. Alves LR, Giacomini MA, Camelo SHH, Laus AM, Leal LA, Goulart BF, et al. Evidências sobre trabalho em equipe na atenção hospitalar. J Health NPEPS. 2016;1(2):246-62.

11. Souza G, Peduzzi M, Silva JAM, Carvalho BG. Teamwork in nursing: restrictedtonursingprofessionalsoraninterprofessionalcollaboration? Rev. Esc. Enferm. USP. 2016:50(4):640-7.

12. Oliveira FMCN, Cunha Ferreira E, Rufino NA, Santos MSS. Educação permanente e qualidade da assistência à saúde: aprendizagem significativa no trabalho da enfermagem. Aquichan. 2011;11(1):48-65.

13. Azevedo IC, Santos Silva GW, Vale LD, Santos QG Cassiano AN, Morais IF, et al. Educação continuada em enfermagem no âmbito da educação permanente em saúde: revisão integrativa de literatura. Saude pesq. 2015;8(1):131-40.

14. Moura GMSS, Inchauspe JAF, Dall'Agnol CM, Magalhães AMM, Hoffmeister LV. Expectationsofthenursing staff in relationtotheleadership. Acta paul. enferm. 2013;26(2):198-204.
15. Ribeiro HCC, Campos LI, Manzo BF, Brito MJM, Alves M. Estudo das não conformidades no trabalho da enfermagem: evidências relevantes para melhoria da qualidade hospitalar. Aquichan. 2014;14(4):582-93.

16. Araujo MT, Velloso ISC, Queiroz CF, Henriques AVB. Dimensionamento de pessoal de enfermagem de uma unidade de clínica médica. Rev. enferm. Cent.-Oeste Min. 2016;6(2):2223-34.

17. Zandomenighi RC, Mouro DL, Oliveira CA, Martins EAP. Intensivecare in hospital emergencyservices: Challenges for nurses. Rev. min. enferm. 2014;18(2):404-14.

18. Souza MFG, Santos ADB, Monteiro Al. O processo de enfermagem na concepção de profissionais de Enfermagem de um hospital de ensino. Rev. bras. enferm. 2013;66(2):167-73.

19. Chernicharo IM, Freitas FDS, Ferreira MA. Representações sociais da humanização do cuidado na concepção de usuários hospitalizados. Saude soc. 2013;22(3):830-9.

20. Chernicharo IM, Freitas FDS, Ferreira MA. Humanização no cuidado de enfermagem: contribuição ao debate sobre a Política Nacional de Humanização. Rev. bras. enferm. 2013;66(4):564-70.

\section{CORRESPONDÊNCIA}

Maristela Oliveira Lara

Departamento de Enfermagem, Universidade Federal dos Vales do Jequitinhonha e Mucuri - UFVJM. Campus JK. Rodovia MGT 367 - Km 586, 5000. Alto da Jacuba, Diamantina - Minas Gerais - Brasil. CEP: 39100-000. E-mail: maryslara@hotmail.com 